\title{
Two-color QCD with non-zero chiral chemical potential
}

\author{
V.V. Braguta, ${ }^{a, b}$ V.A. Goy, ${ }^{c}$ E.M. Ilgenfritz, ${ }^{d}$ A.Yu. Kotov, ${ }^{e, 1}$ A.V. Molochkov, ${ }^{b}$ \\ M. Müller-Preussker ${ }^{f}$ and B. Petersson ${ }^{f}$ \\ ${ }^{a}$ Institute for High Energy Physics NRC "Kurchatov Institute", \\ 142281 Protvino, Russia \\ ${ }^{b}$ Far Eastern Federal University, School of Biomedicine, \\ 690950 Vladivostok, Russia \\ ${ }^{c}$ Far Eastern Federal University, School of Natural Sciences, \\ 690950 Vladivostok, Russia \\ ${ }^{d}$ Joint Institute for Nuclear Research,BLTP, \\ 141980 Dubna, Russia \\ e Institute of Theoretical and Experimental Physics, \\ 117259 Moscow, Russia \\ ${ }^{f}$ Humboldt-Universität zu Berlin, Institut für Physik, \\ 12489 Berlin, Germany \\ E-mail: braguta@itep.ru, vovagoy@gmail.com, ilgenfri@theor.jinr.ru, \\ kotov@itep.ru, molochkov.alexander@gmail.com, \\ mmp@physik.hu-berlin.de, bengt@physik.hu-berlin.de
}

ABSTRACT: The phase diagram of two-color QCD with non-zero chiral chemical potential is studied by means of lattice simulation. We focus on the influence of a chiral chemical potential on the confinement/deconfinement phase transition and the breaking/restoration of chiral symmetry. The simulation is carried out with dynamical staggered fermions without rooting. The dependences of the Polyakov loop, the chiral condensate and the corresponding susceptibilities on the chiral chemical potential and the temperature are presented. The critical temperature is observed to increase with increasing chiral chemical potential.

KeYwords: Lattice QCD, Phase Diagram of QCD

ArXiv EPrint: 1503.06670 


\section{Contents}

1 Introduction 1

2 Details of the simulations 2

3 Results of the calculation 5

4 Discussion and conclusion $\quad 11$

A Ultraviolet divergences in the chiral condensate $\quad 12$

$\begin{array}{ll}\text { B Ultraviolet divergences in the Polyakov loop } & 15\end{array}$

\section{Introduction}

For the vacuum state of QCD as well as for the properties of finite temperature QCD the existence of non-trivial topological excitations is important. Well known are instantons [1] as classical solutions in Euclidean space as tunneling events between topologically different zero-temperature vacua. The role of topology for the solution of the famous $U_{A}(1)$ problem has been recognized very early [2,3]. Presently, the anomalous breaking of the $U_{A}(1)$ symmetry above the deconfinement transition/crossover is under intense investigation in the lattice community (see, e.g., ref. [4]).

It is known by now from lattice QCD at zero temperature that a (fractal) lowdimensional (laminar) topological vacuum structure is discernible at very fine resolution scale [5], while localized instanton-like structures, actually prevailing at an infrared scale, are believed to explain chiral symmetry breaking $[6,7]$.

The gluon fields contributing to the path integral at finite temperature correspondingly may contain calorons $[8,9]$. Adapted to the non-trivial holonomy they have a richer structure (in terms of "dyonic" constituents) than instantons. The changes of this structure at the QCD phase transition are presently still under study [10, 11].

Some time ago the gluonic topological structure and the famous axial anomaly have been proposed to be immediately observable (and controllable) through the generation of $P$ and $C P$ violating domains (violating also translational invariance) in heavy ion collisions $[12,13]$. It has been demonstrated by detailed numerical calculations $[12,14]$ that macroscopic domains of (anti)parallel color-electric and color-magnetic field can emerge in a heavy ion collision creating an increasing chiral imbalance among the quarks which are deconfined due to the high energy density. In this situation, the magnetic field created by the spectator nucleons may initiate a charge separation relative to the reaction plane (parallel to the electro-magnetic field) [15]. The resulting charge asymmetry of quarks would become observable in terms of recombined hadrons (chiral-magnetic effect) [16, 17]. The strength (and particularly the dependence on the collision energy) of this effect has been 
theoretically studied and proposed to be a signal of the transient existence of liberated quarks $[12,13,18]$.

In recent years the dependence of the chiral and deconfinement transitions on the magnetic field has been investigated both in models and ab-initio lattice simulations, see e.g. $[19,20]$. It remains an open question whether the phase transition from quarks to hadrons, i.e. the onset of confinement and chiral symmetry breaking (and vice versa), depends on the chiral imbalance.

In this paper we study the change of the phase structure induced by a chiral chemical potential in an equilibrium lattice simulation. We mimic the topological content (of a $C P$ violating, topologically nontrivial gluonic background in a heavy ion collision event) by inducing a chiral imbalance, which is provided (i.e. frozen) by a non-zero chiral chemical potential. In this setting, the modification of the phase diagram by the chiral chemical potential $\mu_{5}$ has been studied mainly by means of effective models [21-27], the predictions of which will later be compared with our results.

On the lattice, contrary to the case of non-zero baryon chemical potential, simulations with non-vanishing $\mu_{5}$ are not hampered by a sign problem. ${ }^{1}$ Thus, one can employ the standard hybrid Monte Carlo algorithms. Such lattice simulations with $\mu_{5} \neq 0$ were already performed in ref. $[28,29]$. The main goal of these papers, however, was the study of the chiral magnetic effect. Therefore, the phase diagram was not systematically studied.

In refs. [30, 31] we have carried out the first lattice study of the phase diagram with non-zero chiral chemical potential. It was performed in SU(2) QCD with four flavors, which we have considered as a simplified model of QCD. In this article we extend this investigation generating considerably more data and performing a more detailed analysis. But we do not attempt to analyse the topological structure that would reflect the presence of the chiral chemical potential.

One reason for choosing the $\mathrm{SU}(2)$ gauge group is that less computational resources are required for this pilot study than for full QCD. The second reason is that we have already carried out two-colour QCD computations with an external magnetic field [32, 33]. Furthermore, the four flavor case results from our choice of staggered fermions as lattice regularization, while we avoid to take the root of the fermion determinant, which would allow to reduce the number of flavors. The "rooting procedure" might introduce further systematic errors at finite lattice spacing.

In section 2 we introduce the model and its lattice implementation and define the quantities we measure. In section 3 we present our results, and section 4 is devoted to their discussion and to the formulation of conclusions. In the appendix we discuss the question of renormalizations refering to explicit analytical calculations in perturbation theory.

\section{Details of the simulations}

We have performed simulations with the $\mathrm{SU}(2)$ gauge group. We employ the standard Wilson plaquette action

$$
S_{g}=\beta \sum_{x, \mu<\nu}\left(1-\frac{1}{N_{c}} \operatorname{Tr} U_{\mu \nu}(x)\right) .
$$

\footnotetext{
${ }^{1} \mathrm{In} \mathrm{SU}(2)$ there is no sign problem even in presence of a baryon chemical potential.
} 
For the fermionic part of the action we use staggered fermions

$$
\begin{aligned}
S_{f}= & m a \sum_{x} \bar{\psi}_{x} \psi_{x}+ \\
& +\frac{1}{2} \sum_{x \mu} \eta_{\mu}(x)\left(\bar{\psi}_{x+\mu} U_{\mu}(x) \psi_{x}-\bar{\psi}_{x} U_{\mu}^{\dagger}(x) \psi_{x+\mu}\right) \\
& +\frac{1}{2} \mu_{5} a \sum_{x} s(x)\left(\bar{\psi}_{x+\delta} \bar{U}_{x+\delta, x} \psi_{x}-\bar{\psi}_{x} \bar{U}_{x+\delta, x}^{\dagger} \psi_{x+\delta}\right),
\end{aligned}
$$

where the $\eta_{\mu}(x)$ are the standard staggered phase factors: $\eta_{1}(x)=1, \eta_{\mu}(x)=$ $(-1)^{x_{1}+\ldots+x_{\mu-1}}$ for $\mu=2,3,4$. The lattice spacing is denoted by $a$, the bare fermion mass by $m$, and $\mu_{5}$ is the value of the chiral chemical potential. In the chirality breaking term $s(x)=(-1)^{x_{2}}, \delta=(1,1,1,0)$ represents a shift to the diagonally opposite site in a spatial $2^{3}$ elementary cube. The combination of three links connecting sites $x$ and $x+\delta$,

$$
\bar{U}_{x+\delta, x}=\frac{1}{6} \sum_{i, j, k=\operatorname{perm}(1,2,3)} U_{i}\left(x+e_{j}+e_{k}\right) U_{j}\left(x+e_{k}\right) U_{k}(x)
$$

is symmetrized over the 6 shortest paths between these sites. In the partition function, after formally integrating over the fermions, one obtains the corresponding determinant. As mentioned above, we do not take the fourth root of this determinant in order to represent each flavor ("taste") independently of the others. Thus, the continuum limit of our model corresponds to a theory of four (degenerate) flavors.

In the continuum limit eq. (2.2) can be rewritten in the Dirac spinor-flavor basis $[34,35]$ as follows

$$
S_{f} \rightarrow S_{f}^{(\text {cont })}=\int d^{4} x \sum_{i=1}^{4} \bar{q}_{i}\left(\partial_{\mu} \gamma_{\mu}+i g A_{\mu} \gamma_{\mu}+m+\mu_{5} \gamma_{5} \gamma_{4}\right) q_{i} .
$$

We would like to emphasize that the chiral chemical potential, introduced in eq. (2.2), corresponds to the taste-singlet operator $\gamma_{5} \gamma_{4} \otimes \mathbf{1}$ in the continuum limit.

It should be also noted here that the usual baryonic chemical potential after the discussion in ref. [36], and also the chiral chemical potential as used in ref. [29], are introduced to the action as a modification of the temporal links by corresponding exponential factors in order to eliminate chemical-potential dependent quadratic divergences. For staggered fermions this modification can be performed as well for the baryonic chemical potential. However, for the chiral chemical potential such a modification would lead to a highly nonlocal action [29]. Therefore, we decided to introduce $\mu_{5}$ in eq. (2.2) in an additive way similar to the mass term.

It is known that the additive way of introducing the chemical potential leads to additional divergences in observables. In the appendix we present analytical and numerical investigations of additional divergences in the Polyakov loop and the chiral condensate. Our study shows that there is no additional divergence in the Polyakov loop, whereas there is an additional logarithmic divergence in the chiral condensate. The latter is numerically small and does not effect the results of this paper. 
We have performed simulations with two lattice sizes $N_{\tau} \times N_{\sigma}^{3}=6 \times 20^{3}, 10 \times 28^{3}$. The measured observables are

- the Polyakov loop

$$
L=\frac{1}{N_{\sigma}^{3}} \sum_{n_{1}, n_{2}, n_{3}}\left\langle\operatorname{Tr} \prod_{n_{4}=1}^{N_{\tau}} U_{4}\left(n_{1}, n_{2}, n_{3}, n_{4}\right)\right\rangle
$$

- the chiral condensate

$$
a^{3}\langle\bar{\psi} \psi\rangle=-\frac{1}{N_{\tau} N_{\sigma}^{3}} \frac{1}{4} \frac{\partial}{\partial(m a)} \log Z=\frac{1}{N_{\tau} N_{\sigma}^{3}} \frac{1}{4}\left\langle\operatorname{Tr} \frac{1}{D+m a}\right\rangle,
$$

- the Polyakov loop susceptibility

$$
\chi_{L}=N_{\sigma}^{3}\left(\left\langle L^{2}\right\rangle-\langle L\rangle^{2}\right)
$$

- the disconnected part of the chiral susceptibility

$$
\chi_{\text {disc }}=\frac{1}{N_{\tau} N_{\sigma}^{3}} \frac{1}{16}\left(\left\langle\left(\operatorname{Tr} \frac{1}{D+m a}\right)^{2}\right\rangle-\left\langle\operatorname{Tr} \frac{1}{D+m a}\right\rangle^{2}\right) .
$$

The Polyakov loop and the corresponding susceptibility are sensitive to the confinement/deconfinement phase transition, whereas the chiral condensate in principle responds to chiral symmetry breaking/restoration.

The simulations have been carried out with a CUDA code in order to perform the simulations using the Hybrid Monte Carlo algorithm on GPU's.

The parameters of our lattice calculation are the inverse of the bare coupling constant $\beta$, the bare mass $m a$ and the bare chiral chemical potential $a \mu_{5}$ (both in dimensionless units) as well as the lattice size. The physical temperature and the volume are given by

$$
\begin{aligned}
& V=\left(N_{\sigma} a(\beta)\right)^{3} \\
& T=\frac{1}{a(\beta) N_{\tau}} .
\end{aligned}
$$

To perform the scale setting (calibration) of the lattice, we use the results of ref. [32]. There, the static potential in the same theory has been measured at zero temperature for several values of $\beta$. The Sommer parameter $r_{0}$ was calculated in lattice units and compared to its physical value, which was supposed to be the same as in QCD, i.e. $r_{0}=0.468(4) \mathrm{fm}$. It was found that the scaling function $a(\beta)$ in the region were we perform our measurements is well described by the two loop $\beta$-function. Thus, for given $\beta$ we can obtain e.g. the temperature $T$ in units of $\mathrm{MeV}$. For more details, see ref. [32]. 


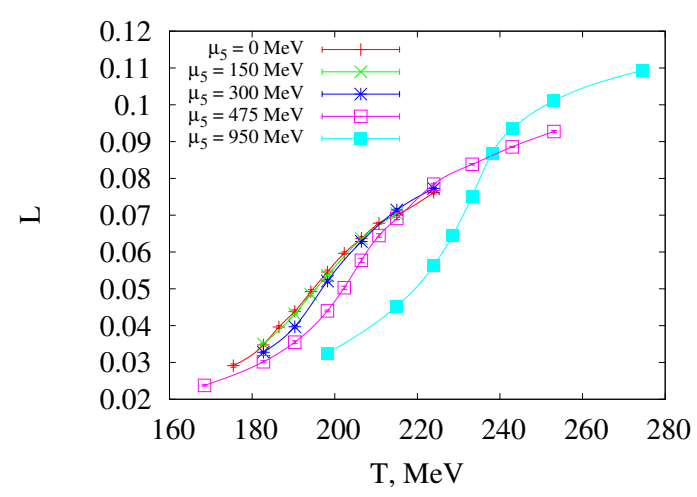

Polyakov loop

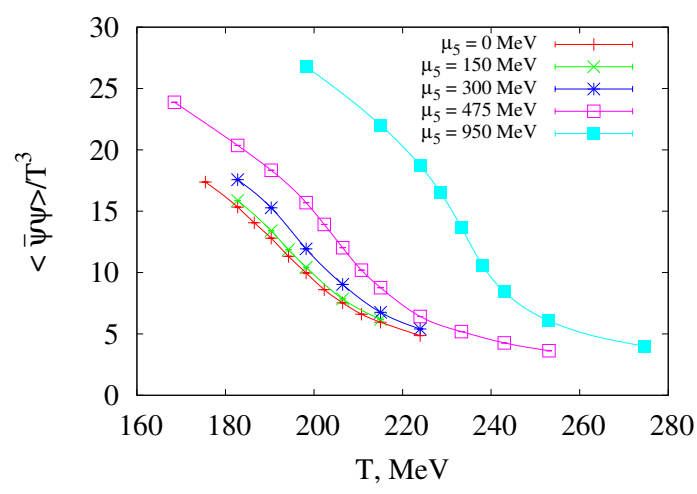

Chiral condensate

Figure 1. Polyakov loop and chiral condensate versus $T$ for five values of $\mu_{5}$. Lattice size is $6 \times 20^{3}$, fermion mass is $m \approx 12 \mathrm{MeV}$. Errors are smaller than the data point symbols. The curves are to guide the eyes.

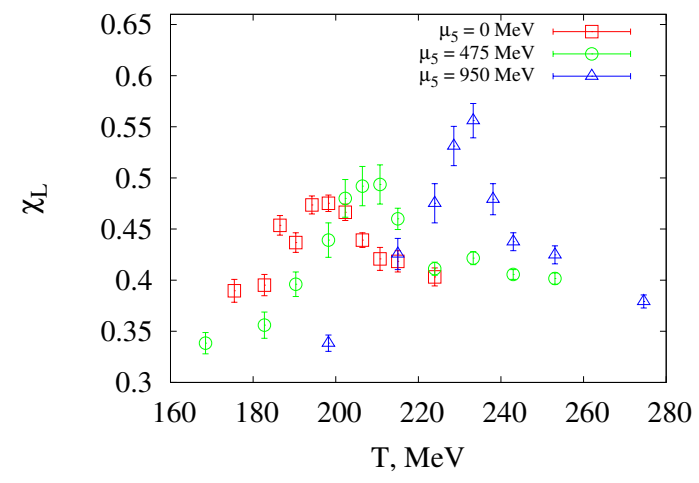

Polyakov loop susceptibility

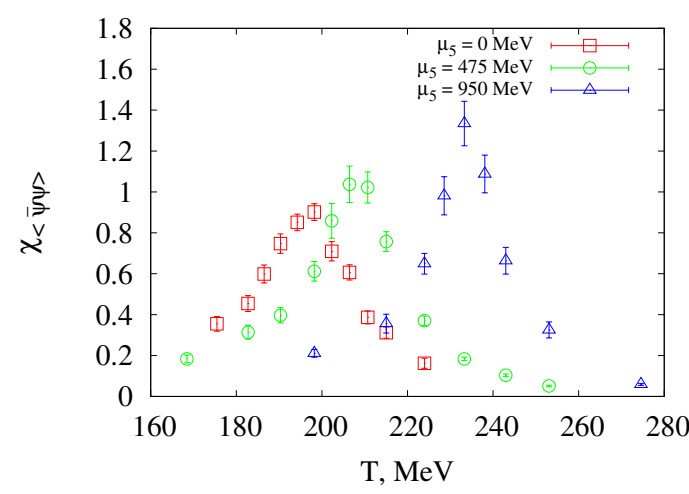

Chiral susceptibility

Figure 2. Polyakov loop susceptibility and chiral susceptibility versus $T$ for three values of $\mu_{5}=$ $0,475,950 \mathrm{MeV}$. Lattice size is $6 \times 20^{3}$, fermion mass is $m \approx 12 \mathrm{MeV}$. In order to avoid a complete superposition of data points belonging to different $\mu_{5}$ values we applied a tiny shift along the $T$ axis.

\section{Results of the calculation}

We first performed simulations on a lattice of size $6 \times 20^{3}$ for five fixed values of $\mu_{5}=$ $0,150,300,475,950 \mathrm{MeV}$ and different values of $T$. The fermion mass was kept fixed in physical units $m \approx 12 \mathrm{MeV}\left(m_{\pi} \approx 330 \mathrm{MeV}\right)$. The expectation values of the Polyakov loop and the chiral condensate are shown in figure 1. The sharp change of the observables as functions of $T$ indicates the onset of the deconfinement and the chiral restoration phase transition. It is seen that the temperature of both phase transitions increases with the chiral chemical potential. One also sees that the phase transition becomes sharper for increasing chiral chemical potential.

To study the change of the critical temperature more quantitatively, we also calculated the chiral and the Polyakov loop susceptibilities. In order to make the figures readable we 


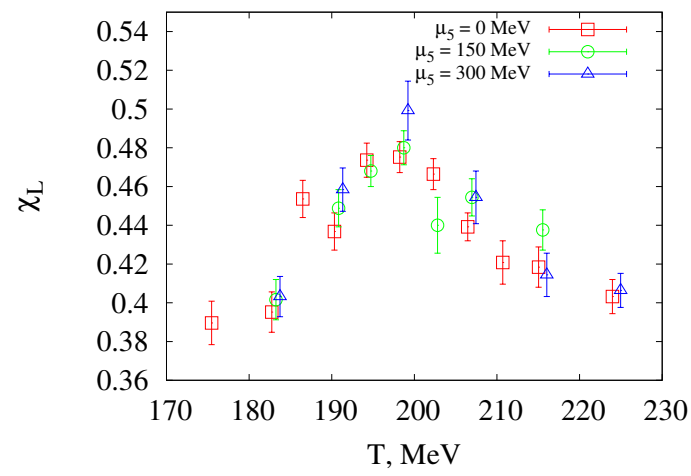

Polyakov loop susceptibility

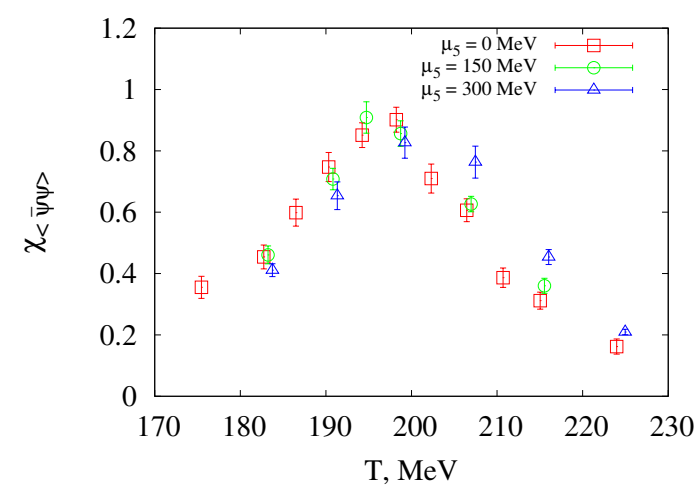

Chiral susceptibility

Figure 3. Same as figure 2 but for the three values of $\mu_{5}=0,150,300 \mathrm{MeV}$.

plot separately the susceptibilities for values $\mu_{5}=0,475,950 \mathrm{MeV}$ in figure 2 and for values $\mu_{5}=0,150,300 \mathrm{MeV}$ in figure 3 . We see that increasing the value of the chiral chemical potential moves the position of the peaks of the chiral and Polyakov loop susceptibilities to larger values of $\beta$. This means that the transition temperature increases. We have fitted the data for both the susceptibilities near the peak with a Gaussian function $\chi=a_{0}+a_{1} \exp \left(-\frac{\left(\beta-\beta_{c}\right)^{2}}{2 \sigma^{2}}\right)$ and extracted the critical temperatures $T_{c}^{\chi}\left(\beta_{c}^{\chi}\right)$ using the chiral susceptibility and $T_{c}^{L}\left(\beta_{c}^{L}\right)$ using the Polyakov loop susceptibility. The resulting dependences of both critical temperatures on the value of the chiral chemical potential $\mu_{5}$ are shown on the figure 4 and table 1 . The values of $\beta_{c}^{\chi}, \beta_{c}^{L}$ and $T_{c}^{\chi}, T_{c}^{L}$ and their uncertainties are calculated from a fit of 5-6 points in the vicinity of the peak by the Gaussian function given above. One sees that for all points except for $\mu_{5}=950 \mathrm{MeV}$ the critical temperatures $T_{c}^{\chi}$ and $T_{c}^{L}$ coincide within errors. There is a slight discrepancy for $\mu_{5}=950 \mathrm{MeV}$, but because of possible systematic uncertainties in the fit as well as finite size effects, we cannot claim that the transition temperatures are different.

It should be noted that for small values of $\mu_{5}$ the dependence of $T_{c}$ on $\mu_{5}$ is well described by the function $T_{c}=a+b \mu_{5}^{2}$, but the larger $\mu_{5}$ is the larger is the deviation from this simple formula.

In addition to the calculations on the $6 \times 20^{3}$ lattice we carried out simulations on a larger lattice of size $10 \times 28^{3}$. Note that this allows us to investigate larger values of $\mu_{5}$. The susceptibilities require large statistics, and therefore our current computational resources do not allow us to measure them on the larger lattice. In the simulations we kept the physical fermion mass fixed at $m=18.5 \mathrm{MeV}\left(m_{\pi} \approx 550 \mathrm{MeV}\right)$.

On this lattice we also calculated the observables $L$ and $\langle\bar{\psi} \psi\rangle$ as functions of $T$ for different values of $\mu_{5}$. The results for the Polyakov loop and the chiral condensate are presented in figure 5. They underpin the fact that the critical temperature grows when one increases $\mu_{5}$. The value of the chiral chemical potential used in our simulations was rather large (up to $\mu_{5}=3345 \mathrm{MeV}$ ), but we have not seen signals of a first-order phase transition, although the transition becomes sharper when the value of the chiral chemical potential grows. 


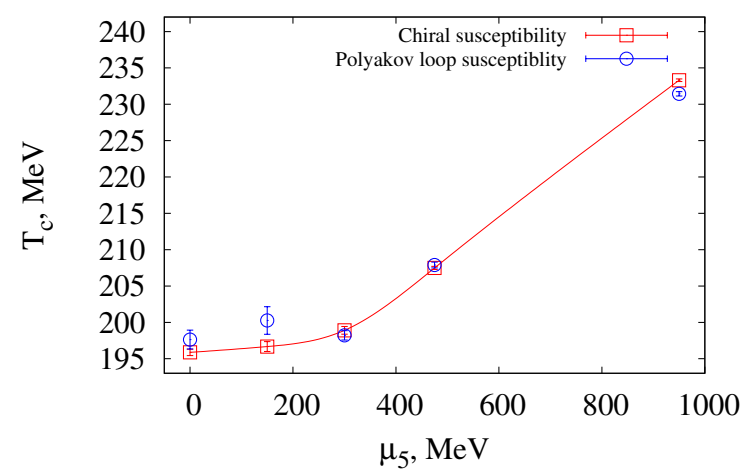

Figure 4. The dependence of the critical temperatures $T_{c}^{\chi}$ and $T_{c}^{L}$ on the value of the chiral chemical potential. Lattice size is $6 \times 20^{3}$, fermion mass is $m \approx 12 \mathrm{MeV}$. The curve connecting the points for $T_{c}^{\chi}$ is to guide the eyes.

\begin{tabular}{|c|c|c|c|c|}
\hline$\mu_{5}(\mathrm{MeV})$ & $\beta_{c}^{\chi}$ & $T_{c}^{\chi}(\mathrm{MeV})$ & $\beta_{c}^{L}$ & $T_{c}^{L}(\mathrm{MeV})$ \\
\hline 0 & $1.7975 \pm 0.0005$ & $195.8 \pm 0.4$ & $1.7993 \pm 0.0016$ & $197.6 \pm 1.3$ \\
150 & $1.7984 \pm 0.0009$ & $196.7 \pm 0.7$ & $1.8025 \pm 0.0023$ & $200.3 \pm 1.9$ \\
300 & $1.8012 \pm 0.0007$ & $198.9 \pm 0.5$ & $1.8000 \pm 0.0008$ & $198.2 \pm 0.6$ \\
475 & $1.8116 \pm 0.0003$ & $207.5 \pm 0.2$ & $1.8117 \pm 0.0005$ & $207.9 \pm 0.4$ \\
950 & $1.8404 \pm 0.0002$ & $233.3 \pm 0.2$ & $1.8381 \pm 0.0003$ & $231.4 \pm 0.3$ \\
\hline
\end{tabular}

Table 1. The critical temperatures $T_{c}^{\chi}$ and $T_{c}^{L}$ and the lattice parameters $\beta_{c}^{\chi}$ and $\beta_{c}^{L}$ as a function of the chiral chemical potential $\mu_{5}$ obtained from the fit of the chiral and Polyakov loop susceptibilities near the peak by a Gaussian function $\chi=a_{0}+a_{1} \exp \left(-\frac{\left(\beta-\beta_{c}\right)^{2}}{2 \sigma^{2}}\right)$.

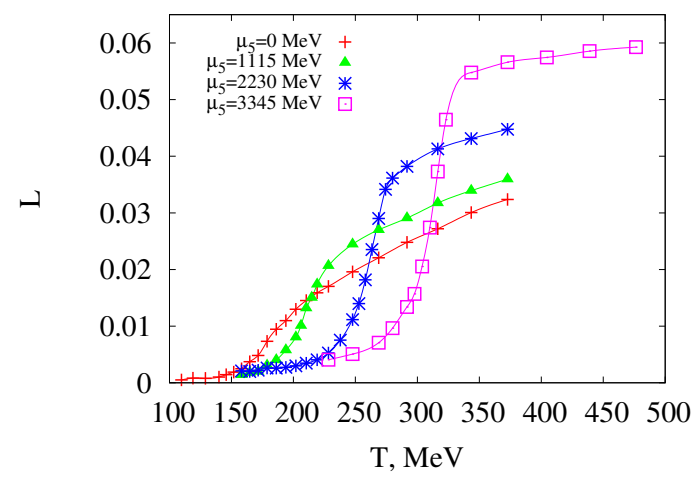

Polyakov loop

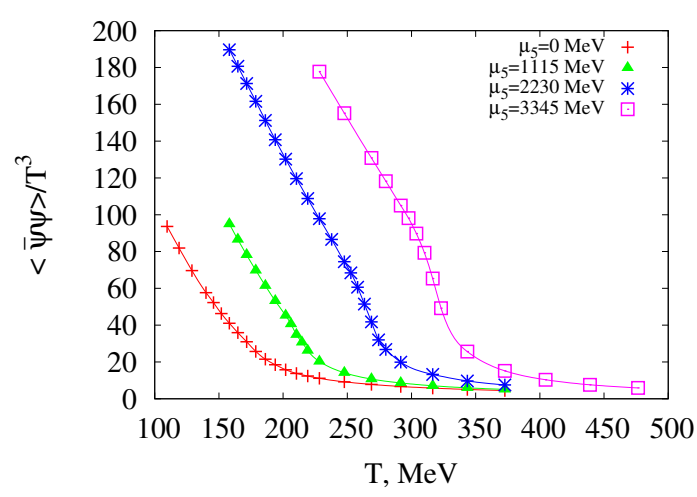

Chiral condensate

Figure 5. Polyakov loop and chiral condensate versus $T$ for four values of $\mu_{5}$ and lattice size $10 \times 28^{3}$, fermion mass is $m a \approx 18.5 \mathrm{MeV}$. Errors are smaller than the data point symbols. The curves are to guide the eyes. 


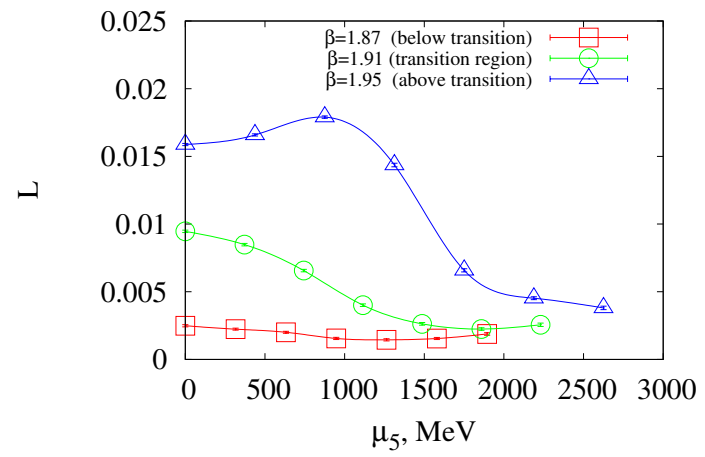

Polyakov loop

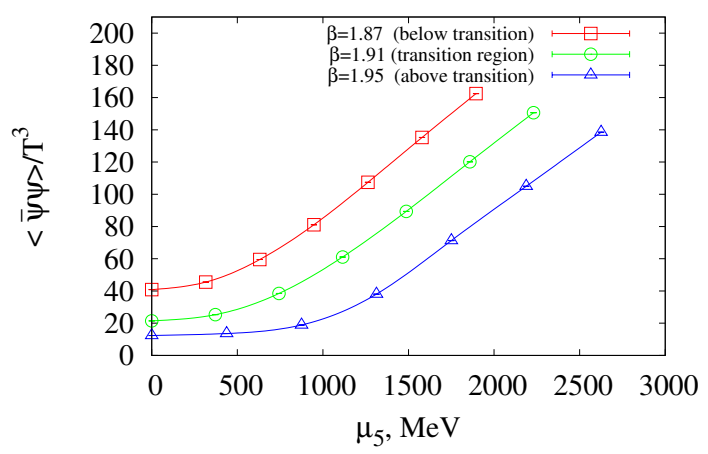

Chiral condensate

Figure 6. Polyakov loop and chiral condensate versus $\mu_{5}$ for three $\beta$ values and lattice size $10 \times 28^{3}$, fermion mass is $m a \approx 18.5 \mathrm{MeV}$. Errors are smaller than the data point symbols. The curves are to guide the eyes.
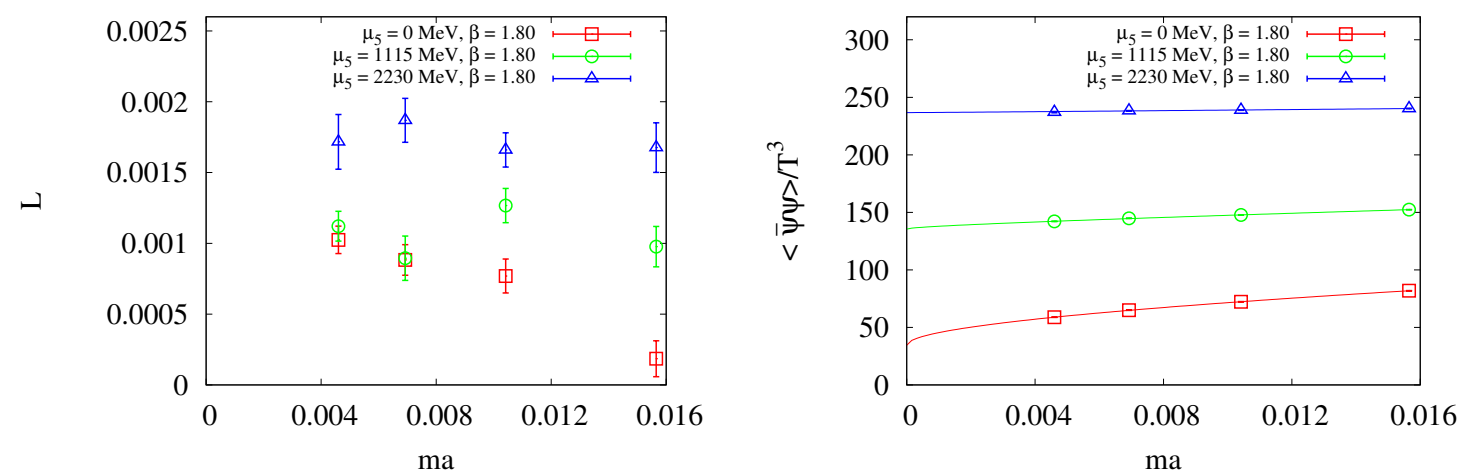

Figure 7. Polyakov loop and chiral condensate versus $m a$ for three $\mu_{5}$ values at $\beta=1.80$ on a lattice of size $10 \times 28^{3}$. $\beta=1.80$ corresponds to the confinement phase.

It is interesting to study how the observables $L$ and $\langle\bar{\psi} \psi\rangle$ depend on $\mu_{5}$ for fixed temperature. To do this we have measured the Polyakov loop and the chiral condensate as functions of $\mu_{5} a$ for three different values of $\beta, \beta=1.87(158 \mathrm{MeV}), 1.91(186 \mathrm{MeV})$ and $1.95(219 \mathrm{MeV})$, which for a vanishing chiral chemical potential corresponds to a temperature below the transition, in the transition region, and in the high temperature phase, respectively. The results of these measurements are shown in figure 6. As can be seen from the figure, in the confinement phase the Polyakov loop remains almost constant with increasing chiral chemical potential. It means that if the system was in the confinement phase at $\mu_{5}=0$, it remains confined at $\mu_{5}>0$. Moreover, we observe the Polyakov loop to drop down with increasing $\mu_{5}$ both in the deconfinement phase and in the transition region. Thus, the system goes into the confinement phase for sufficiently large $\mu_{5}$. With other words, we conclude that the critical temperature increases with an increasing chiral chemical potential in agreement with our results obtained on the smaller lattice. It is worth mentioning that the behavior described above looks quite similar to the behavior obtained for two-color QCD in an external magnetic field [10, 32]. 


\begin{tabular}{|c|c|c|c|c|c|c|c|c|c|}
\hline \multicolumn{2}{|c|}{} & \multicolumn{9}{|c|}{$f_{1}(m a)$} & \multicolumn{4}{c|}{$f_{2}(m a)$} \\
\hline$\beta$ & $\mu_{5}, \mathrm{MeV}$ & $\chi_{\text {dof }}^{2}$ & $a_{0}$ & $a_{1}$ & $a_{2}$ & $\chi_{\text {dof }}^{2}$ & $b_{0}$ & $b_{1}$ & $b_{2}$ \\
\hline 1.80 & 0 & 0.56 & $0.034(1)$ & $0.34(2)$ & $0.3(1)$ & 1.2 & $0.042(1)$ & $-0.9(1)$ & $-1.1(4)$ \\
1.80 & 1115 & 1.83 & $0.135(2)$ & $0.06(5)$ & $0.6(2)$ & 2.0 & $0.137(1)$ & $-0.15(13)$ & $0.4(5)$ \\
1.80 & 2230 & 2.0 & $0.237(5)$ & $-0.004(100)$ & $0.3(5)$ & 2.0 & $0.237(3)$ & $0.02(26)$ & $0.3(9)$ \\
1.90 & 1115 & 2.7 & $0.034(3)$ & $0.31(6)$ & $0.3(3)$ & 3.9 & $0.042(2)$ & $-0.8(2)$ & $-1.0(7)$ \\
1.90 & 2230 & 0.62 & $0.144(2)$ & $0.18(3)$ & $-0.11(15)$ & 0.3 & $0.1486(5)$ & $-0.47(6)$ & $-0.9(2)$ \\
\hline
\end{tabular}

Table 2. The parameters for the fits $f_{1}(m a)$ eq. (3.1) and $f_{2}(m a)$ eq. (3.2) allowing to extrapolate to the chiral limit for $\beta=1.80$ and $\beta=1.90$ and various values of chiral chemical potential. The fit curves obtained with $f_{1}$ are shown in the right panel of figure 7 and figure 8 .
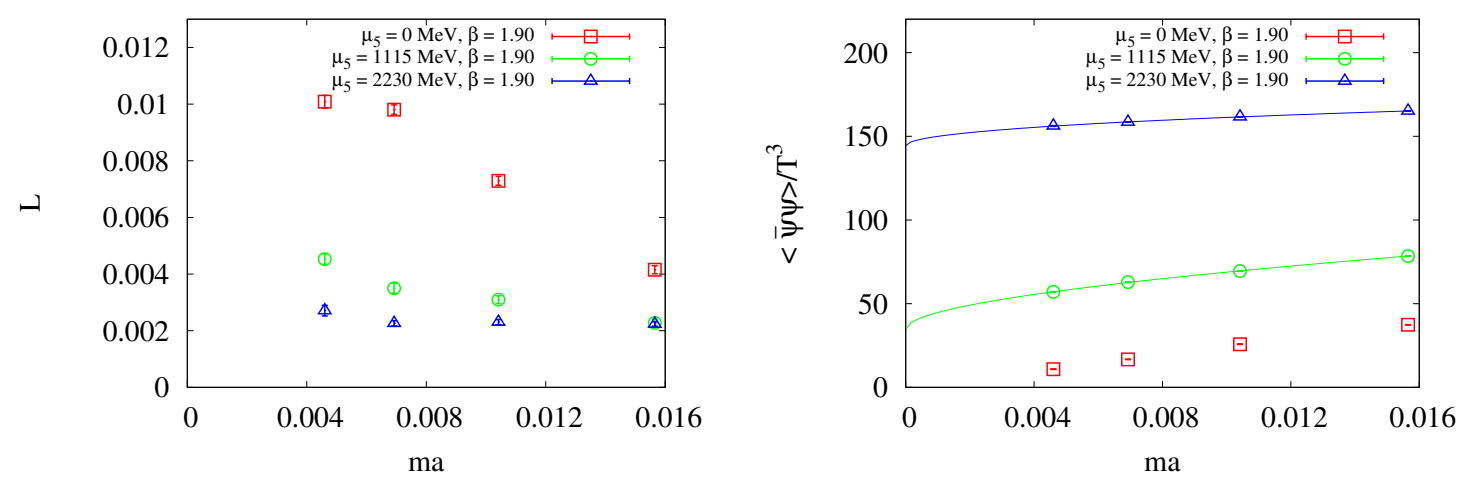

Figure 8. Same as figure 7 , but for $\beta=1.90$ corresponding to the transition region.

In order to study the dependence of the observables on the value of the quark mass for different values of chiral chemical potential, we have chosen four values of $\beta$ : one in the confinement phase, $\beta=1.8$ (corresponding to a temperature $T=119 \mathrm{MeV}$ ), two in the transition region, $\beta=1.9(T=178 \mathrm{MeV})$ and $\beta=2.0(T=268 \mathrm{MeV})$, and one in the deconfinement phase $\beta=2.1(T=404 \mathrm{MeV})$. The lattice size used here is $10 \times 28^{3}$. We have taken four values of the fermion mass $m a$ and three values of the chemical potential $\mu_{5}=0 \mathrm{MeV}, \mu_{5}=1115 \mathrm{MeV}$ and $\mu_{5}=2230 \mathrm{MeV}$. The expectation values of the Polyakov loop and the chiral condensate are presented as functions of $m a$ for different $\beta$ in figure 7 , figure 8, figure 9, and figure 10 .

In figure 7 we show the results in the confinement region at $\beta=1.80(T=119 \mathrm{MeV})$. The Polyakov loop is small and does not show any nontrivial behavior. The chiral condensate remains almost constant when the value of the fermion mass changes.

To extrapolate to the chiral limit $(m a=0)$ we performed chiral extrapolations in two ways. Since the value of the temperature is not very far from the transition temperature, we suppose that we can use the formula for the reduced three dimensional model [37]. See also [38-40]. In that case we may use the ansatz $a^{3}<\bar{\psi} \psi>=f_{1}(m a)$, where

$$
f_{1}(m a)=a_{0}+a_{1} \sqrt{m a}+a_{2} m a
$$

with the non-analytic term coming from the Goldstone bosons. Such a parametrization 

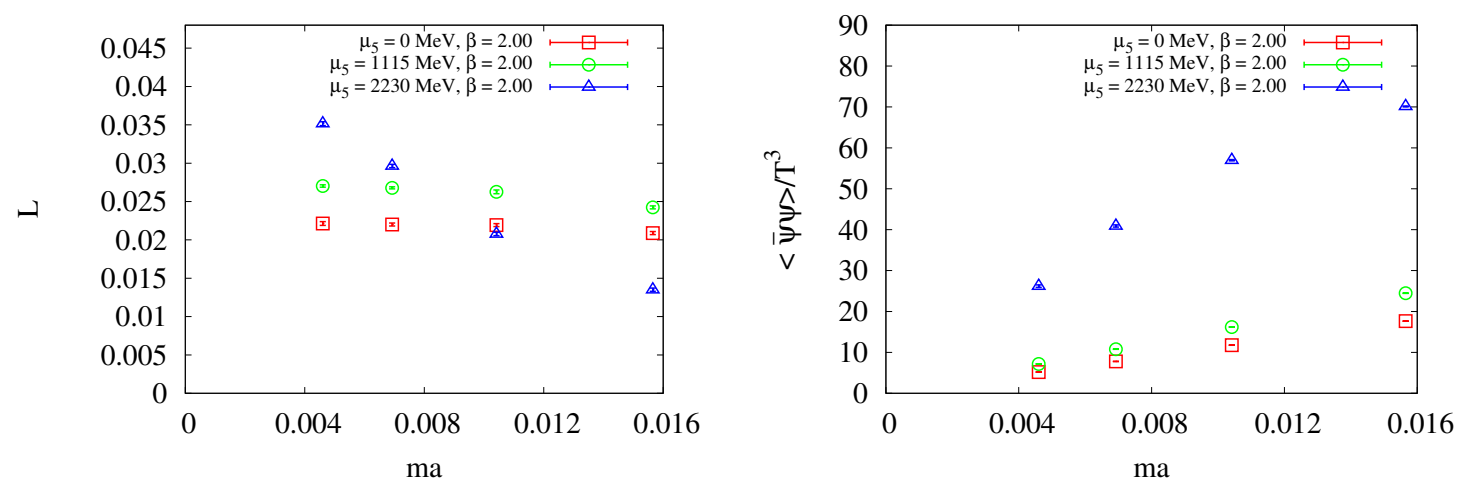

Figure 9. Same as figure 7, but for $\beta=2.00$ corresponding to the transition region as well.
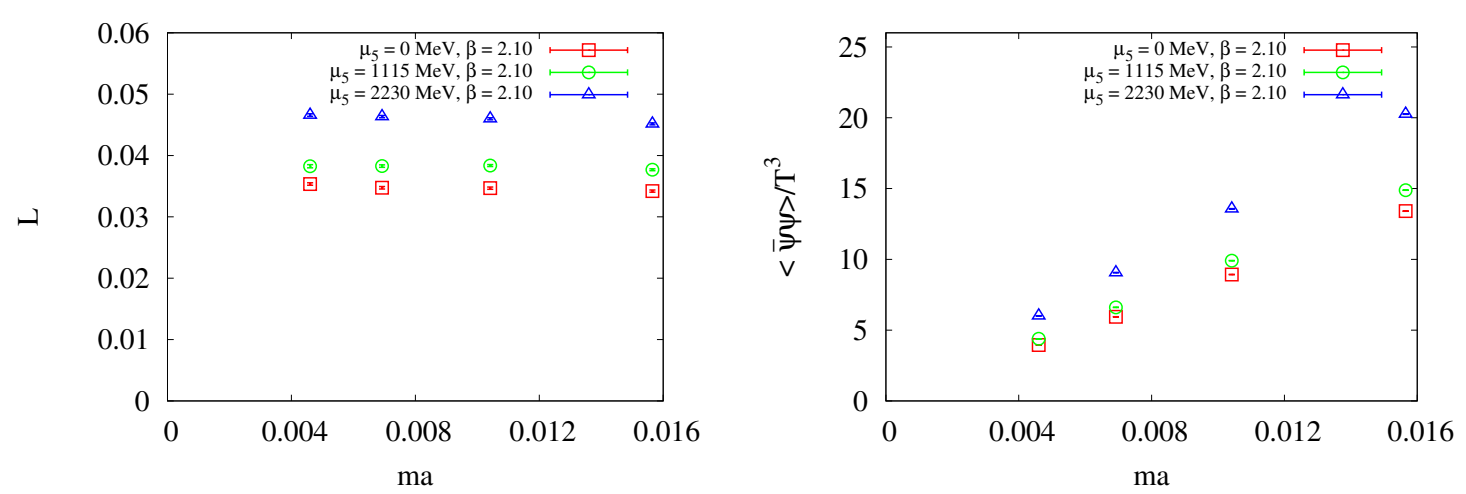

Figure 10. Same as figure 7, but for $\beta=2.10$ corresponding to the deconfinement phase.

has been used in [41] in the context of the finite temperature transition in QCD. As an alternative we also use the chiral extrapolation relevant for zero temperature, namely $a^{3}<\bar{\psi} \psi>=f_{2}(m a)$, where

$$
f_{2}(m a)=b_{0}+b_{1} m a \log m a+b_{2} m a .
$$

The fits performed with the eq. (3.1) are shown as lines in figure 7. The corresponding parameters of both are given in table 2. As can be seen from this table and the corresponding figure, the chiral condensate extrapolates to a non-zero value in the chiral limit.

At larger values $\beta=1.90(T=178 \mathrm{MeV}), \beta=2.00(T=268 \mathrm{MeV})$, and $\beta=2.10$ $(T=404 \mathrm{MeV})$ and at zero chiral chemical potential the system is in the chirally restored phase, when $m$ goes to zero. However, at $\beta=1.90(T=178 \mathrm{MeV})$ and $\mu_{5}=1115,2230 \mathrm{MeV}$, the chiral condensate has non-zero expectation values. We have performed the same extrapolation technique as for $\beta=1.80$ and present also these results in table 2 , showing that for these values of $\mu_{5}$ the data are consistent with non-zero values in the chiral limit. It implies that also in the chiral limit a non-zero chiral chemical potential shifts the position of the phase transition to larger temperatures. The plot for the Polyakov loop (left panel of figure 8) confirms this observation: for the two smaller values of the fermion mass $m a$ the values of Polyakov loop for $\mu_{5} a=0$ is several times larger than 
for non-zero values of $\mu_{5}$, so that the system for non-zero $\mu_{5}$ is deeper in the confinement region than for zero chiral chemical potential.

At $\beta=2.00(T=268 \mathrm{MeV})$ and $\beta=2.10(T=404 \mathrm{MeV})$ the chiral condensate goes to zero in the chiral limit (figure 9,10 ), thus the system is in the chirally restored phase. The dependence of the chiral condensate on the value of the mass is almost a linear function, apart from the behavior for $\beta=2.00(T=268 \mathrm{MeV})$ at the largest chiral chemical potential under our investigation, $\mu_{5}=2230 \mathrm{MeV}$. This behavior can be explained if one assumes that this point is near the phase transition. It is known that increasing the mass shifts the position of the transition to larger temperatures [32]. Thus, at smaller masses the system is in the chirally restored phase, while increasing the mass lets the system undergo the chiral phase transition. Again, the plot of the Polyakov loop confirms this suggestion. The Polyakov loop is small at larger masses implying that the system is in the confinement phase and increases when the mass is decreased, i.e. the system becomes deconfining. This behavior suggests that at the largest value of $\mu_{5}=2230 \mathrm{MeV}$ the transition happens at even larger temperature than we have investigated. These results are in total agreement with the results described above.

\section{Discussion and conclusion}

In this paper we have presented an investigation of the phase diagram of two-color QCD with a chiral chemical potential using lattice simulations with dynamical staggered fermions without rooting, i.e. with four flavor degrees of freedom in the continuum limit.

We have calculated the chiral condensate and the Polyakov loop for different values of the temperature $T$ and chiral chemical potential $\mu_{5}$ on lattices of size $6 \times 20^{3}$ and $10 \times 28^{3}$ as well as their respective susceptibilities (for the smaller lattice size). Our main result is the observation that the finite temperature phase transition becomes clearly shifted to larger critical temperature with rising chiral chemical potential. It was seen that this conclusion remains true when one is extrapolating to vanishing quark mass. Additionally by comparing the peak positions of the Polyakov loop susceptibility with those of the chiral susceptibility we saw the respective critical temperatures $T_{c}^{L}$ and $T_{c}^{\chi}$ to agree within errors at least up to $\mu_{5} \approx 0.5 \mathrm{GeV}$. For larger $\mu_{5}$ values we were not able to draw a final conclusion about the (dis)agreement.

Our result is in contradiction to results obtained with various effective models of QCD [21-23, 26, 27], where the critical temperature was said to decrease as $\mu_{5}$ increases.

We concede that we study two-color QCD with $N_{f}=4$ quarks which is different from what is mostly considered in the effective models. For a closer comparison, it would be very interesting to examine the corresponding model case or to carry out the corresponding lattice simulation in the $\mathrm{SU}(3)$ case.

It is likely, however, that the contradictions are rather a consequence of the fact that the critical temperature is not a universal parameter but crucially depends on the structure and parameters of the effective models. It is unclear to what extent they describe the actual behavior of finite temperature QCD. It should be also noted that some predictions obtained in different effective models are in conflict with each other. For instance, the dependence of the chiral condensate on $\mu_{5}$ obtained in papers [21, 22] is opposite to the result obtained 
in paper [25]. The latter paper claims that the chiral condensate grows with the chiral chemical potential, which is in agreement with our results.

In addition to the dependence of the critical temperature on the chiral chemical potential, some effective models predict that - beginning from some critical value of $\mu_{5}^{c}$ ( $\mu_{5}^{c} \approx 400 \mathrm{MeV}$ in ref. [21], $\mu_{5}^{c}=50 \mathrm{MeV}$ in ref. [22]) - the transition from the hadronic to the plasma phase turns into a first order transition. In our simulations we see that the transition becomes sharper as we increase $\mu_{5}$, but we don't see a first order phase transition up to $\mu_{5} \approx 3.3 \mathrm{GeV}$, which would manifest itself as discontinuity in the Polyakov loop and chiral condensate at a sufficiently large spatial extension of the lattice. In this connection a finite size scaling analysis would be valuable but is outside the scope of this paper.

Besides within effective models, the phase diagram of QCD in the $\left(\mu_{5}, T\right)$-plane was studied in papers $[42,43]$ in a framework of Dyson-Schwinger equations. The authors of these papers found that the critical temperature rises with $\mu_{5}$ and the "phase transition" actually is always a crossover. These results are corroborating the results of our paper.

We would like also to mention the paper [44]. In this paper the authors address the question of universality of phase diagrams in QCD and QCD-like theories through the large- $N_{c}$ equivalence. Using the results of this paper one can show that at large $N_{c}$ and in the chiral limit there is an equivalence between the QCD phase diagram at finite $\mu_{5}$ and the QCD phase diagram at finite isospin chemical potential $\mu_{I}$. The chiral condensate of QCD with $\mu_{5} \neq 0$ can be mapped to the pion condensate of QCD with $\mu_{I} \neq 0$. In the latter theory the pion condensate and the critical temperature $T_{c}$ of pion condensation increase with $\mu_{I}$. Despite the fact that we considered $N_{c}=2$ one can expect that the chiral condensate and $T_{c}$ in our theory also increase with $\mu_{5}$. We believe this is one more fact in favor of our results.

\section{Acknowledgments}

The authors are grateful to V.I. Zakharov and V.G. Bornyakov for interesting and stimulating discussions. The authors are grateful to N. Yamamoto who directed our attention to universality in large- $N_{c}$ QCD-like theories. The simulations were performed at GPUs of the K100 supercomputer of the Institute of Applied Mathematics of the Russian Academy of Sciences in Moscow and at GPUs of the Particle Phenomenology Group at the Institute of Physics of the Humboldt University Berlin. The work was supported by Far Eastern Federal University, by RFBR grants 13-02-01387-a, 14-02-01185-a, 15-02-07596-a and 1532-21117, by a grant of the president of the RF, MD-3215.2014.2, and by a grant of the FAIR-Russia Research Center. The work of A. Yu. Kotov was also partially supported by the Dynasty foundation.

\section{A Ultraviolet divergences in the chiral condensate}

The fermion propagator including the chiral chemical potential for "naive" lattice fermions can be written in the following form

$$
S^{\alpha \beta}(x, y)=\frac{\delta^{\alpha \beta}}{L_{t} L_{s}^{3}} \sum_{\{p\}} \sum_{s} e^{i p(x-y)} \frac{-i \sum_{\mu} \gamma_{\mu} \sin \left(p_{\mu}\right)+m+\mu_{5} \gamma_{0} \gamma_{5}}{\sin ^{2}\left(p_{0}\right)+\left(|p|-s \mu_{5}\right)^{2}+m^{2}} \times P(s),
$$




$$
\begin{aligned}
P(s) & =\frac{1}{2}\left(1-i s \sum_{i} \frac{\gamma_{i} \sin \left(p_{i}\right)}{|p|} \gamma_{0} \gamma_{5}\right), \quad i=1,2,3, \\
|p|^{2} & =\sin ^{2}\left(p_{1}\right)+\sin ^{2}\left(p_{2}\right)+\sin ^{2}\left(p_{3}\right), \\
p_{i} & =\frac{2 \pi}{L_{s}} n_{i}, \quad i=1,2,3, \quad n_{i}=0, \ldots, L_{s}-1, \\
p_{4} & =\frac{2 \pi}{L_{t}} n_{4}+\frac{\pi}{L_{t}}, \quad n_{4}=0, \ldots, L_{t}-1 .
\end{aligned}
$$

Here $m$ and $\mu_{5}$ are mass and chiral chemical potential in lattice units, $\alpha, \beta$ are color indices, the sum is taken over all possible values of $\left(n_{1}, n_{2}, n_{3}, n_{4}\right)$ and $s= \pm 1$.

In the limit $L_{s}, L_{t} \rightarrow \infty$ the condensate per one fermion flavor can be written as

$$
\langle\bar{\psi} \psi\rangle=\frac{1}{16} \operatorname{Tr}[S(x, x)]=\frac{m}{4} \int_{-\pi}^{\pi} \frac{d^{4} p}{(2 \pi)^{4}} \sum_{s} \frac{1}{\sin ^{2}\left(p_{0}\right)+\left(|p|-s \mu_{5}\right)^{2}+m^{2}} .
$$

To calculate the integral in formula (A.2) we use the standard approach, which separates the main divergence from the rest of the integral

$$
\begin{aligned}
\int_{-\pi}^{\pi} \frac{d^{4} p}{(2 \pi)^{4}} \sum_{s} \frac{1}{\sin ^{2}\left(p_{0}\right)+\left(|p|-s \mu_{5}\right)^{2}+m^{2}}=\int_{-\pi}^{\pi} \frac{d^{4} p}{(2 \pi)^{4}} \frac{2}{\sin ^{2}\left(p_{0}\right)+|p|^{2}+m^{2}} \\
\quad+\int_{-\pi}^{\pi} \frac{d^{4} p}{(2 \pi)^{4}} \sum_{s}\left(\frac{1}{\sin ^{2}\left(p_{0}\right)+\left(|p|-s \mu_{5}\right)^{2}+m^{2}}-\frac{1}{\sin ^{2}\left(p_{0}\right)+|p|^{2}+m^{2}}\right) .
\end{aligned}
$$

The first term in this expression is just the loop integral for $\langle\bar{\psi} \psi\rangle$ without chiral chemical potential. The expression for this integral in the continuum limit $a \rightarrow 0$ can be found in $[45]$

$$
\int_{-\pi}^{\pi} \frac{d^{4} p}{(2 \pi)^{4}} \frac{1}{\sin ^{2}\left(p_{0}\right)+|p|^{2}+m^{2}}=0.619734+m^{2}\left(\frac{\log \left(m^{2}\right)}{\pi^{2}}-0.345071\right)+O\left(m^{4}\right) .
$$

The second term in (A.3) is proportional to $\mu_{5}^{2}$ and contains a logarithmic divergence in the continuum limit. This divergence can be calculated using saddle-point method. To calculate the second term in (A.3) we subtract the leading divergence and calculate the rest of the integral in the limit $a \rightarrow 0$. The result of the calculation can be written as follows

$$
\begin{array}{r}
\int_{-\pi}^{\pi} \frac{d^{4} p}{(2 \pi)^{4}} \sum_{s}\left(\frac{1}{\sin ^{2}\left(p_{0}\right)+\left(|p|-s \mu_{5}\right)^{2}+m^{2}}-\frac{1}{\sin ^{2}\left(p_{0}\right)+|p|^{2}+m^{2}}\right) \\
=\mu_{5}^{2}\left(-4 \frac{\log \left(m^{2}\right)}{\pi^{2}}+0.671036\right)+O\left(m^{4}, \mu_{5}^{2} m^{2}, \mu_{5}^{4}\right) .
\end{array}
$$

We have checked the last formula numerically. Introducing a physical mass $m_{p} a=m$ and a physical chiral chemical potential $\mu_{5}^{p} a=\mu_{5}$ one can write the expression for the condensate in physical units as

$$
\langle\bar{\psi} \psi\rangle_{p}=0.309867 \frac{m_{p}}{a^{2}}+m_{p}^{3}\left(\frac{\log \left(m_{p} a\right)}{\pi^{2}}-0.172536\right)
$$




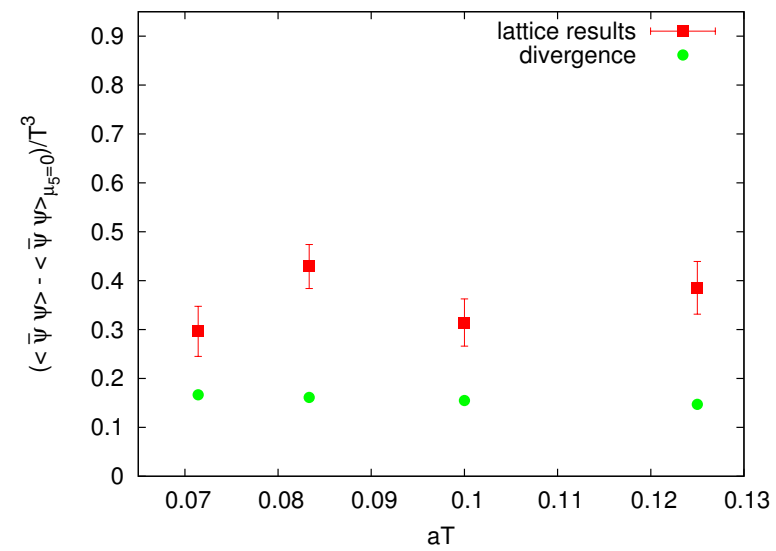

Figure 11. The difference between chiral condensates at $\mu_{5}=0 \mathrm{MeV}$ and $\mu_{5}=330 \mathrm{MeV}$ for different lattice spacings $(T=270 \mathrm{MeV})$. Square points correspond to lattice measurements, circle points correspond to the contribution of logarithmic divergence (A.6) to the difference.

$$
+m_{p}\left(\mu_{5}^{p}\right)^{2}\left(-2 \frac{\log \left(m_{p} a\right)}{\pi^{2}}+0.167759\right)
$$

From the last formula one sees that at one-loop level the inclusion of a non-zero chiral chemical potential leads to an additional logarithmic divergence. We believe that this conclusion persists if one takes into account radiative corrections to formula (A.6). To see this, consider some Feynman graph of radiative corrections to formula (A.6). The expansion of this graph in powers of $\mu_{5}$ can be reduced to the inclusion of a dimension- 3 operator to the graph that diminishes the power of divergence by one unit per one power of $\mu_{5}$. Expansion of the chiral condensate in powers of $\mu_{5}$ contains only even powers. The main divergence of the chiral condensate is $\sim 1 / a^{2}$. So, the next to leading term in $\mu_{5}$ expansion is two powers smaller than $\sim 1 / a^{2}$, i.e. it is at most logarithmically divergent.

Further it is important to understand how the additional divergence connected to nonzero $\mu_{5}$ effects the results of this paper. To estimate this we fixed the physical values of temperature $T=270 \mathrm{MeV}$, quark mass $m_{p}=33 \mathrm{MeV}$ and measured the condensate $\langle\bar{\psi} \psi\rangle$ in the deconfinement phase for $\mu_{5}=0$ and $\mu_{5}=330 \mathrm{MeV}$ for different values of lattice spacing $a$. In particular, we took the following lattice parameters: $16^{3} \times 8 \beta=1.9500$, $20^{3} \times 10 \beta=2.0047,24^{3} \times 12 \beta=2.0493$ and $28^{3} \times 14 \beta=2.0870$. The difference between the measured chiral condensates at zero and non-zero $\mu_{5}$ for different lattice spacings is shown in figure 11. In addition to the lattice measurement, in figure 11 we plot the contribution of logarithmic divergence (A.6) to the difference $\langle\bar{\psi} \psi\rangle-\langle\bar{\psi} \psi\rangle_{\mu_{5}=0}$.

From figure 11 one sees that the uncertainty of the calculation does not allow to confirm the logarithmic type of the divergence. However, the variation of the lattice results with variation of the lattice spacing is very slow what allows us to assume that there are no other divergences different from the logarithmic one. Note also that the value of the $\mu_{5} \neq 0$ contribution to the chiral condensate obtained from the one-loop expression for the logarithmic divergence is by a factor 2-3 smaller than the lattice results. The difference can be attributed to a non-zero temperature effect and radiative corrections. This fact allows 


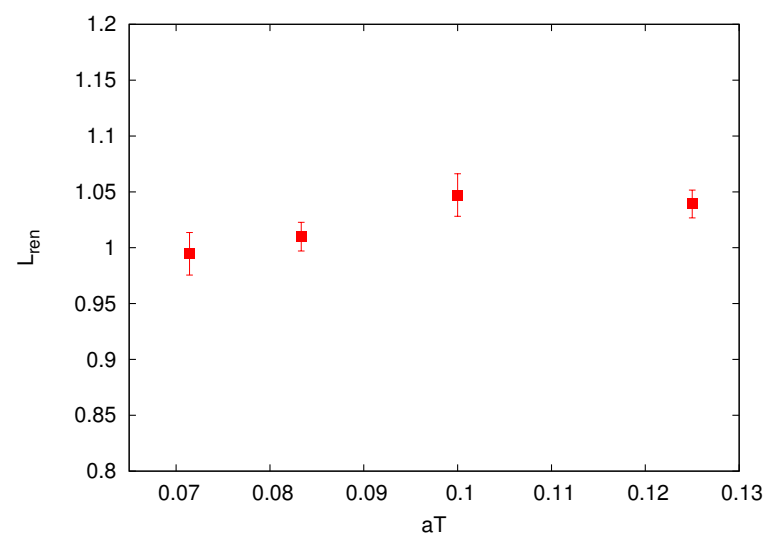

Figure 12. Renormalized Polyakov loop (B.4) as a function of the lattice spacing ( $T=270 \mathrm{MeV})$.

one to state that the renormalization effect of the one-loop expression for the logarithmic divergence (A.6) is not very large. So one can use it to estimate the contribution of the ultraviolet logarithmic divergence in the confinement phase.

The calculation shows that the characteristic values of the difference $\langle\bar{\psi} \psi\rangle-\langle\bar{\psi} \psi\rangle_{\mu_{5}=0}$ in the confinement phase for different values of lattice parameters used in this paper are approximately by one order of magnitude larger than the additional ultraviolet logarithmic divergence due to $\mu_{5} \neq 0$ estimated according to formula (A.6). For instance, from figure 1 one can see that at temperature $T=200 \mathrm{MeV}$ (close to the phase transition at $\mu_{5}=0$ ) the difference $\left(\langle\bar{\psi} \psi\rangle_{\mu_{5}=300 \mathrm{MeV}}-\langle\bar{\psi} \psi\rangle_{\mu_{5}=0}\right) / T^{3} \approx 2$. At the same time the additional ultraviolet divergence according to formula (A.6) gives $\left(\langle\bar{\psi} \psi\rangle_{\text {add }}\right) / T^{3} \approx 0.1$. Note also that the position of the phase transition manifests itself as a peak of the susceptibility. Evidently, there is no peak due to the additional ultraviolet divergence. All this allows us to state that the conclusions obtained in this paper are not affected by the additional ultraviolet $\mu_{5} \neq 0$ divergence in the chiral condensate.

\section{B Ultraviolet divergences in the Polyakov loop}

At the leading order approximation in the strong coupling constant $g$ the Polyakov loop can be written in the following form

$$
L=1-\beta \frac{g^{2}}{4} \int_{0}^{\beta} d \tau D_{00}^{a a}(\tau, \overrightarrow{0})=1-\beta \frac{g^{2}}{4} \int \frac{d^{3} q}{(2 \pi)^{3}} \tilde{D}_{00}^{a a}(0, \vec{q})
$$

where $D_{00}^{a b}(t, \vec{x})$ and $\tilde{D}_{00}^{a a}\left(q_{0}, \vec{q}\right)$ represent the propagator of the temporal components of the gluon field in coordinate and momentum space, correspondingly, $\beta$ is inverse temperature, and the summation over color index $a$ is assumed. From eq. (B.1) it is clear that additional divergences connected to non-zero $\mu_{5}$ at one-loop level can appear from the fermion selfenergy part of the gluon propagator. The study of the divergences with "naive" fermions in the self-energy part of the propagator is cumbersome. For this reason we use momentum cut of regularization procedure to study the divergences in the Polyakov loop. 
The fermion contribution to the one-loop self-energy of the gluon propagator $\tilde{D}_{00}^{a b}$ can be written in the following form

$$
\begin{aligned}
\Pi_{00}(\vec{q})= & \frac{1}{4} \sum_{s_{1}, s_{2}} \int^{\Lambda} \frac{d^{4} k}{(2 \pi)^{4}} \frac{-k_{0}^{2}+\left(\mu_{5}-s_{1}|\vec{k}|\right)\left(\mu_{5}-s_{2}|\vec{k}+\vec{q}|\right)+m^{2}}{\left(k_{0}^{2}+\left(|\vec{k}|-s_{1} \mu_{5}\right)^{2}+m^{2}\right)\left(k_{0}^{2}+\left(|\vec{k}+\vec{q}|-s_{2} \mu_{5}\right)^{2}+m^{2}\right)} \times \\
& \times\left(1+s_{1} s_{2} \frac{\vec{k} \cdot(\vec{k}+\vec{q})}{|\vec{k}||\vec{k}+\vec{q}|}\right),
\end{aligned}
$$

where $\vec{q}$ is the external momentum, $s_{1}, s_{2}= \pm 1$.

To proceed we expand equation (B.2) in $\mu_{5}^{2}$. Evidently each new term in this expansion diminishes the power of divergence by two units. That means that beginning from $\mu_{5}^{4}$ term the series is ultraviolet convergent. The first term $\left(\left.\Pi_{00}(\vec{q})\right|_{\mu_{5}=0}\right)$ is just the expression for the self-energy without chiral chemical potential. So, to calculate the divergence which results from nonzero $\mu_{5}$ one needs to study only the divergence of the second term. Taking the derivative of equation (B.2) with respect to $\mu_{5}^{2}$ we get

$$
\begin{aligned}
\Pi_{00}(\vec{q})-\left.\Pi_{00}(\vec{q})\right|_{\mu_{5}=0}=4 \mu_{5}^{2} \int \frac{d^{4} k}{(2 \pi)^{4}} & \left(\frac{1}{\left(k_{0}^{2}+|\vec{k}|^{2}+m^{2}\right)\left(k_{0}^{2}+|\vec{k}+\vec{q}|^{2}+m^{2}\right)}\right. \\
& -\frac{2 m^{2}+4|k+q|^{2}+6 \vec{k} \cdot(\vec{k}+\vec{q})-2 k_{0}^{2}}{\left(k_{0}^{2}+|\vec{k}|^{2}+m^{2}\right)^{2}\left(k_{0}^{2}+|\vec{k}+\vec{q}|^{2}+m^{2}\right)} \\
& +\frac{8|\vec{k}|^{2}\left(m^{2}+\vec{k} \cdot(\vec{k}+\vec{q})-k_{0}^{2}\right)}{\left(k_{0}^{2}+|\vec{k}|^{2}+m^{2}\right)^{3}\left(k_{0}^{2}+|\vec{k}+\vec{q}|^{2}+m^{2}\right)} \\
& \left.+\frac{4\left(m^{2}-k_{0}^{2}\right)(\vec{k} \cdot(\vec{k}+\vec{q}))+4|\vec{k}|^{2}|\vec{k}+\vec{q}|^{2}}{\left(k_{0}^{2}+|\vec{k}|^{2}+m^{2}\right)^{2}\left(k_{0}^{2}+|\vec{k}+\vec{q}|^{2}+m^{2}\right)^{2}}\right)+O\left(\mu_{5}^{4}\right) .
\end{aligned}
$$

One can easily check that this expression is free from ultraviolet divergence. Moreover, a calculation shows that at large $\vec{q}^{2}$ expression (B.3) behaves as $\Pi_{00}(\vec{q})-\left.\Pi_{00}(\vec{q})\right|_{\mu_{5}=0} \sim$ $\mu_{5}^{2}\left(c_{1} \log \vec{q}^{2}+c_{2}\right)$, where $c_{1}, c_{2}$ are some constants. So, at one-loop level there is no ultraviolet divergence in the Polyakov loop which results from non-zero chiral chemical potential. Similarly to the previous section, one can argue that there is no ultraviolet divergence in the $\mu_{5} \neq 0$ contribution to the Polyakov loop at higher loops.

In order to numerically study the role of divergences in the Polyakov loop due to non-zero chiral chemical potential, we used the following definition of renormalized Polyakov loop

$$
L_{\mathrm{ren}}=\frac{L}{L_{\mu_{5}=0}},
$$

where $L$ is the Polyakov loop and $L_{\mu_{5}=0}$ is the Polyakov loop at the same lattice but with $\mu_{5}=0$. Evidently $L_{r e n}$ doesn't contain divergences that are usual for simulations with zero chiral chemical potential. Similarly to the previous section we fixed the physical values of temperature $T=270 \mathrm{MeV}$, quark mass $m_{p}=33 \mathrm{MeV}$ and measured the $L_{r e n}$ in the deconfinement phase for $\mu_{5}=330 \mathrm{MeV}$ and different values of lattice spacing $a$. The result of this measurement is shown in figure 12 
From this figure one sees that the renormalized Polyakov loop $L_{r e n}$ is consistent with a constant in the region of lattice spacings investigated. In the same region the unrenormalized Polyakov loop $L$ changes by a factor of two. From these facts we conclude that there is no additional ultraviolet divergence in Polyakov loop due to non-zero chiral chemical potential.

Open Access. This article is distributed under the terms of the Creative Commons Attribution License (CC-BY 4.0), which permits any use, distribution and reproduction in any medium, provided the original author(s) and source are credited.

\section{References}

[1] A.A. Belavin, A.M. Polyakov, A.S. Schwartz and Y. Tyupkin, Pseudoparticle solutions of the Yang-Mills equations, Phys. Lett. B 59 (1975) 85 [INSPIRE].

[2] E. Witten, Current algebra theorems for the U(1) 'Goldstone boson', Nucl. Phys. B 156 (1979) 269 [INSPIRE].

[3] G. Veneziano, U(1) without instantons, Nucl. Phys. B 159 (1979) 213 [InSPIRE].

[4] V. Dick, F. Karsch, E. Laermann, S. Mukherjee and S. Sharma, Microscopic origin of $U_{A}(1)$ symmetry violation in the high temperature phase of QCD, Phys. Rev. D 91 (2015) 094504 [arXiv: 1502.06190] [inSPIRE].

[5] E.-M. Ilgenfritz et al., Vacuum structure revealed by over-improved stout-link smearing compared with the overlap analysis for quenched QCD, Phys. Rev. D 77 (2008) 074502 [arXiv:0801.1725] [INSPIRE].

[6] T. Schäfer and E.V. Shuryak, Instantons in QCD, Rev. Mod. Phys. 70 (1998) 323 [hep-ph/9610451] [INSPIRE].

[7] E.V. Shuryak and T. Schäfer, Instantons and chiral symmetry restoration in QCD-like theories, Nucl. Phys. Proc. Suppl. 53 (1997) 472 [inSPIRE].

[8] T.C. Kraan and P. van Baal, Periodic instantons with nontrivial holonomy, Nucl. Phys. B 533 (1998) 627 [hep-th/9805168] [INSPIRE].

[9] K.-M. Lee and C.-h. Lu, SU(2) calorons and magnetic monopoles, Phys. Rev. D 58 (1998) 025011 [hep-th/9802108] [INSPIRE].

[10] E.-M. Ilgenfritz, B.V. Martemyanov and M. Müller-Preussker, Topology near the transition temperature in lattice gluodynamics analyzed by low lying modes of the overlap Dirac operator, Phys. Rev. D 89 (2014) 054503 [arXiv: 1309.7850] [INSPIRE].

[11] V.G. Bornyakov, E.M. Ilgenfritz, B.V. Martemyanov and M. Muller-Preussker, Dyon structures in the deconfinement phase of lattice gluodynamics: topological clusters, holonomies and Abelian monopoles, Phys. Rev. D 91 (2015) 074505 [arXiv: 1410.4632] [INSPIRE].

[12] D. Kharzeev, Parity violation in hot QCD: Why it can happen and how to look for it, Phys. Lett. B 633 (2006) 260 [hep-ph/0406125] [INSPIRE].

[13] K. Fukushima, D.E. Kharzeev and H.J. Warringa, The chiral magnetic effect, Phys. Rev. D 78 (2008) 074033 [arXiv:0808.3382] [INSPIRE]. 
[14] D. Kharzeev, A. Krasnitz and R. Venugopalan, Anomalous chirality fluctuations in the initial stage of heavy ion collisions and parity odd bubbles, Phys. Lett. B 545 (2002) 298 [hep-ph/0109253] [INSPIRE].

[15] D.E. Kharzeev, L.D. McLerran and H.J. Warringa, The effects of topological charge change in heavy ion collisions: 'Event by event P and CP-violation', Nucl. Phys. A 803 (2008) 227 [arXiv: 0711.0950] [INSPIRE].

[16] STAR collaboration, B.I. Abelev et al., Azimuthal charged-particle correlations and possible local strong parity violation, Phys. Rev. Lett. 103 (2009) 251601 [arXiv:0909.1739] [INSPIRE].

[17] STAR collaboration, B.I. Abelev et al., Observation of charge-dependent azimuthal correlations and possible local strong parity violation in heavy ion collisions, Phys. Rev. C 81 (2010) 054908 [arXiv:0909.1717] [INSPIRE].

[18] STAR collaboration, L. Adamczyk et al., Beam-energy dependence of charge separation along the magnetic field in Au+Au collisions at RHIC, Phys. Rev. Lett. 113 (2014) 052302 [arXiv: 1404.1433] [INSPIRE].

[19] I.A. Shovkovy, Magnetic catalysis: a review, Lect. Notes Phys. 871 (2013) 13 [arXiv: 1207.5081] [INSPIRE].

[20] M. D'Elia, Lattice QCD simulations in external background fields, Lect. Notes Phys. 871 (2013) 181 [arXiv: 1209.0374] [INSPIRE].

[21] K. Fukushima, M. Ruggieri and R. Gatto, Chiral magnetic effect in the PNJL model, Phys. Rev. D 81 (2010) 114031 [arXiv: 1003.0047] [InSPIRE].

[22] M.N. Chernodub and A.S. Nedelin, Phase diagram of chirally imbalanced QCD matter, Phys. Rev. D 83 (2011) 105008 [arXiv:1102.0188] [INSPIRE].

[23] R. Gatto and M. Ruggieri, Hot quark matter with an axial chemical potential, Phys. Rev. D 85 (2012) 054013 [arXiv: 1110.4904] [INSPIRE].

[24] A.A. Andrianov, D. Espriu and X. Planells, Chemical potentials and parity breaking: the Nambu-Jona-Lasinio model, Eur. Phys. J. C 74 (2014) 2776 [arXiv:1310.4416] [INSPIRE].

[25] X. Planells, A.A. Andrianov, V.A. Andrianov and D. Espriu, An effective theory for QCD with an axial chemical potential, PoS (QFTHEP 2013) 049 [arXiv:1310.4434] [INSPIRE].

[26] J. Chao, P. Chu and M. Huang, Inverse magnetic catalysis induced by sphalerons, Phys. Rev. D 88 (2013) 054009 [arXiv:1305.1100] [INSPIRE].

[27] L. Yu, H. Liu and M. Huang, Spontaneous generation of local CP-violation and inverse magnetic catalysis, Phys. Rev. D 90 (2014) 074009 [arXiv:1404.6969] [INSPIRE].

[28] A. Yamamoto, Chiral magnetic effect in lattice QCD with a chiral chemical potential, Phys. Rev. Lett. 107 (2011) 031601 [arXiv:1105.0385] [INSPIRE].

[29] A. Yamamoto, Lattice study of the chiral magnetic effect in a chirally imbalanced matter, Phys. Rev. D 84 (2011) 114504 [arXiv:1111.4681] [inSPIRE].

[30] V. Braguta et al., Study of the phase diagram of $\mathrm{SU}(2)$ quantum chromodynamics with nonzero chirality, JETP Lett. 100 (2015) 547.

[31] V.V. Braguta et al., Two-color QCD with chiral chemical potential, PoS (LATTICE2014) 235 [arXiv: 1411.5174] [INSPIRE]. 
[32] E.-M. Ilgenfritz, M. Kalinowski, M. Muller-Preussker, B. Petersson and A. Schreiber, Two-color QCD with staggered fermions at finite temperature under the influence of a magnetic field, Phys. Rev. D 85 (2012) 114504 [arXiv:1203.3360] [INSPIRE].

[33] E.M. Ilgenfritz, M. Muller-Preussker, B. Petersson and A. Schreiber, Magnetic catalysis (and inverse catalysis) at finite temperature in two-color lattice QCD, Phys. Rev. D 89 (2014) 054512 [arXiv: 1310.7876] [INSPIRE].

[34] H. Kluberg-Stern, A. Morel, O. Napoly and B. Petersson, Flavors of lagrangian Susskind fermions, Nucl. Phys. B 220 (1983) 447 [InSPIRE].

[35] I. Montvay and G. Münster, Quantum fields on a lattice, Cambridge University Press, Cambridge U.K. (1994).

[36] P. Hasenfratz and F. Karsch, Chemical potential on the lattice, Phys. Lett. B 125 (1983) 308 [INSPIRE].

[37] R.D. Pisarski and F. Wilczek, Remarks on the chiral phase transition in chromodynamics, Phys. Rev. D 29 (1984) 338 [InSPIRE].

[38] D.J. Wallace and R.K.P. Zia, On singularities induced by goldstone modes, Phys. Rev. B 12 (1975) 5340 [InSPIRE].

[39] P. Hasenfratz and H. Leutwyler, Goldstone boson related finite size effects in field theory and critical phenomena with $O(N)$ symmetry, Nucl. Phys. B 343 (1990) 241 [INSPIRE].

[40] A.V. Smilga and J. Stern, On the spectral density of Euclidean Dirac operator in QCD, Phys. Lett. B 318 (1993) 531 [INSPIRE].

[41] A. Bazavov et al., The chiral and deconfinement aspects of the QCD transition, Phys. Rev. D 85 (2012) 054503 [arXiv: 1111.1710] [INSPIRE].

[42] B. Wang, Y.L. Wang, Z.F. Cui and H.S. Zong, Effect of the chiral chemical potential on the position of the critical endpoint, Phys. Rev. D 91 (2015) 034017.

[43] S.S. Xu et al., Chiral phase transition with a chiral chemical potential in the framework of Dyson-Schwinger equations, Phys. Rev. D 91 (2015) 056003.

[44] M. Hanada and N. Yamamoto, Universality of phase diagrams in QCD and QCD-like theories, PoS (LATTICE 2011) 221 [arXiv:1111.3391] [INSPIRE].

[45] S. Capitani, Lattice perturbation theory, Phys. Rept. 382 (2003) 113 [hep-lat/0211036] [INSPIRE]. 